\title{
Problems with the hydroxyapatite orbital implant: experience with 250 consecutive cases
}

\author{
Carol L Shields, Jerry A Shields, Patrick De Potter, Arun D Singh
}

\begin{abstract}
The coral derived hydroxyapatite sphere is a popular, integrated orbital implant designed to provide improved motility of the ocular prosthesis following enucleation. Although the implant has rapidly become widely used by ophthalmologists, little information is available regarding the problems of this technique in a large series of cases. Experience with 250 consecutive cases of hydroxyapatite orbital implant use was reviewed and the problems of the implants and their management investigated specifically. The reasons for enucleation included uveal melanoma (157 cases), retinoblastoma ( 70 cases), blind painful eye ( 22 cases), and intraocular medulloepithelioma (one case). Earlier treatment to the eye was performed before enucleation in 47 cases and included repair of ruptured globe (17 cases), plaque radiotherapy (18 cases), external beam radiotherapy (six cases), and others (six cases). During a mean of 23 months' follow up (range 6-40 months), there have been no recognisable cases of orbital haemorrhage related to the implant, and no cases of implant extrusion or implant migration. There was one case of presumed orbital infection (culture negative) that resolved with intravenous antibiotics and the implant was retained within the orbit. Other problems included conjunctival thinning in eight cases managed by observation and prosthesis adjustment, and conjunctival erosion in four cases managed by combinations of scleral patch graft, conjunctival flap, and prosthesis adjustment. The conjunctival erosion was caused by a poorly fitting prosthesis in three cases and wound dehiscence in one case. The problem rate in eyes receiving prior radiotherapy or surgery was not increased. The hydroxyapatite integrated orbital implant is a well tolerated motility implant without the high rate of extrusion and infection seen with other motility implants. The prosthesis fit may contribute to the tolerance of the implant.

(Brf Ophthalmol 1994; 78: 702-706)
\end{abstract}

Oncology Service, Wills Eye Hospital, Thomas Jefferson University, Philadelphia, USA C L Shields J A Shields

P De Potter

A D Singh

Correspondence to:

Carol L Shields, MD

Oncology Service, Wills Eye

Hospital, 900 Walnut Street,

Philadelphia, PA 19107,

USA.

Accepted for publication

11 May 1994
Integrated orbital implants are designed to provide improved motility of the ocular prosthesis. ${ }^{1}$ This is accomplished by connecting the moving orbital implant powered by the extraocular muscles to the relatively stationery overlying prosthesis. Historically, there has been a general hesitancy by ophthalmic surgeons to use an integrated orbital implant after enucleation because it often became infected or extruded. Such problems with integrated orbital implants were common with the exposed implants that were used in the $1940 \mathrm{~s}$ and $1950 \mathrm{~s} .{ }^{1-3}$ In order to allow direct integration of the implant with the overlying prosthesis, a portion of the anterior surface of these implants was purposely not covered by human tissue. Unfortunately this exposed portion of the implant served as a site for tissue infection, tissue erosion, and eventual implant extrusion. It was learned from these experiences that an integrated orbital implant should be buried, not exposed. It should be covered completely by conjunctiva so as to prevent the unacceptable complications of the earlier implants.

The hydroxyapatite orbital implant is a buried orbital implant that can be integrated to the overlying prosthesis. ${ }^{4-8}$ It was devised by Perry and associates who studied the performance of the implant initially in animals and later obtained approval by the Food and Drug Administration for use in humans in $1989 .{ }^{5}$ To obtain maximal prosthesis, the implant can be connected to the prosthesis by a peg that is placed into a conjunctival lined orifice in the vascularised buried implant. ${ }^{9}$ The vascularisation of the implant is encouraged by its totally porous framework and is important in preventing infection and extrusion. This is a tremendous improvement over the integrated orbital implants that were used in the past.

We report our experience with 250 consecutive cases of hydroxyapatite orbital implant placed after enucleation. We emphasise the problems of this procedure and their management.

\section{Patients and methods}

We reviewed the charts and examined prospectively all our patients who had a hydroxyapatite orbital implant placed after enucleation. We identified 250 consecutive patients who had the implant placed since we first employed it in January 1990 up to the end of the study period, December 1992. The implant was used only for patients who had enucleation and was not used after evisceration. The selection of patients suitable for the hydroxyapatite orbital implant has been discussed by us previously. ${ }^{\text {? }}$

The conditions for implant evaluated in this study included ocular diagnosis before enucleation, reason for enucleation, and prior ocular surgery or ocular treatment. The size and shape of the hydroxyapatite implant and the use and preparation of the sclera covering of the implant in each case were recorded. Early and late postoperative problems and their management were recorded. Those patients who elected to have the placement of the peg had prior gadolinium-DPTA enhanced magnetic resonance imaging to evaluate precisely the degree of implant vascularity. ${ }^{10}$ Early and late postoperative problems after drilling were recorded and their treatments were evaluated. 
Our technique of enucleation and hydroxyapatite implantation has been recently described ${ }^{7}$ and is similar to other published techniques. ${ }^{71}$ We generally use a $20 \mathrm{~mm}$ sphere in adults and children older than 36 months and we use an $18 \mathrm{~mm}$ sphere in young children. In infants less than 6 months old we usually employ a $16 \mathrm{~mm}$ implant. We soak the brittle hydroxyapatite sphere (Integrated Orbital Implants, San Diego, CA, USA) in balanced salt solution and then wrap it with fresh or banked sclera that has been screened for infectious disease, cancer, and others using the same protocol that is used nationally for corneal donor tissue. The sclera is pretreated with antibiotics and $10 \%$ povidone iodine (Betadine)..$^{7213}$ It is tightly wrapped around the hydroxyapatite sphere and sutured with 5-0 polyglactin 910 (Vicryl) suture, leaving a 12-14 $\mathrm{mm}$ round opening in the sclera at the site of the posterior aspect of the implant. Four rectangular scleral windows $(6 \mathrm{~mm} \times 5 \mathrm{~mm})$ are fashioned for anatomical insertion of the rectus muscles to allow fibrovascular ingrowth at these sites. The scleralised implant is then soaked in antibiotic solution until it is placed in the orbit.

After complete orbital haemostasis, the prepared implant is inserted into the muscle cone of the orbit and all six extraocular muscles are attached to their anatomical sites on the scleralised implant as described. ${ }^{7}$ A thick bed of Tenon's fascia is closed over the implant so that the implant is deeply buried in the mid to posterior orbit. The conjunctiva is closed with a running absorbable suture. A large conformer is placed, antibiotic ointment is instilled, and a heavy pressure dressing is worn for at least 24 hours. The patient is fitted with a standard prosthesis 3 to 6 weeks after the surgical procedure.

Four to six months after the implant is placed, the patient may maximise motility of the prosthesis by undergoing placement of a connecting peg. The orbital tissue is imaged with gadolinium-DTPA enhanced magnetic resonance imaging to assess fibrovascular ingrowth into the implant. ${ }^{10}$ If ingrowth is adequate then the implant is drilled with a $10 \mathrm{~mm} \times 3 \mathrm{~mm}$ bit on the hand drill. The drill bit is aimed perpendicular to the anterior plane of the orbit so that it is anteroposterior in the orbit. A temporary peg is inserted and 3 to 4 weeks later this is replaced with a permanent smooth peg. ${ }^{14}$ The back surface of the prosthesis is then reshaped by an ocularist to conform exactly to the peg contour. A ball and socket connection of the peg to the prosthesis is made and the prosthesis rests on the peg and moves directly with the peg.

\section{Results}

There were 250 consecutive patients in this prospective study who had enucleation and hydroxyapatite implantation. We had no cases of evisceration in our series. There was one case of a secondary implant and the remaining 249 cases were primary implants. The mean follow up was 23 months (median 17 months, range 6-40 months). The clinical reasons for enucleation included uveal melanoma in 157 cases, retinoblastoma in 70 cases, blind painful eye in 22 cases, and medulloepithelioma in one case. There were no cases of enucleation for endophthalmitis. The cause of the blind painful eye included complications of extensive trauma in 12 cases, neovascular glaucoma in four cases, large uveal metastases non-responsive to conservative measures in two cases, intraocular invasion of squamous cell carcinoma of the conjunctiva in one case, and massive tuberculoma of the uvea and sclera with dehiscence of the sclera in one case. ${ }^{15}$ In two of the eyes with uveal melanoma, there was massive extraocular extension that required lateral orbitotomy and modified exenteration removing the globe and the pseudoencapsulated extraocular tumour. ${ }^{16}$ The extraocular muscles and peripheral orbital tissue were preserved and a hydroxyapatite sphere was successfully employed.

Forty seven of the patients had earlier treatment before enucleation, which included shielded plaque radiotherapy in 18 cases, external beam radiotherapy to the globe and orbit in six cases, multiple surgical repairs for a traumatised ruptured globe in 17 cases, partial lamellar sclerouvectomy for a uveal tumour in five cases, and multiple conjunctival excisions for conjunctival squamous cell carcinoma in one case (Table 1). It is calculated that the orbital tissue receives 0 to $200 \mathrm{cGy}$ from a properly shielded iodine-125 plaque with radiation doses used for uveal melanoma. The orbital tissue received 4000 to $5000 \mathrm{cGy}$ in those eyes treated with external beam radiotherapy. The surgical technique was minimally modified as needed for those patients who had earlier surgery. The patients who had prior procedures (including irradiation) on the affected eye experienced no excessive problems and they tolerated the implant well.

In all cases the implant was wrapped in sclera that was pretreated with antibiotics and povidone iodine. We did not use irradiated sclera. The implant was spherical in all cases and was not decapitated anteriorly or reshaped in any way. In children less than 6 months old we used a $16 \mathrm{~mm}$ implant depending on the orbital volume, in children 6 months to 3 years old we used an $18 \mathrm{~mm}$ implant, and in patients older than 3 years we used a $20 \mathrm{~mm}$ implant. We did not use a $22 \mathrm{~mm}$ implant in any case.

Table 1 Earlier treatment to the eye/orbit in 250 cases of hydroxyapatite orbital implantation

\begin{tabular}{ll}
\hline Treatment & Number \\
\hline Plaque radiotherapy & 18 \\
Ruptured globe repair (multiple operations) & 17 \\
External beam radiotherapy & 6 \\
Partial lamellar sclerouvectomy & 5 \\
Conjunctival tumour resection & 1 \\
\hline
\end{tabular}

Table 2 Reasons for and against peg placement in 250 cases of hydroxyapatite orbital implant

\begin{tabular}{lll}
\hline Reason & $\begin{array}{l}\text { No peg } \\
\text { placement } \\
(n=219)\end{array}$ & $\begin{array}{l}\text { Peg } \\
\text { placement } \\
(n=31)\end{array}$ \\
\hline Improved motility & - & 31 \\
Satisfied with motility & 109 & - \\
Patient age $<6$ years & 70 & - \\
Implant age $<6$ months & 37 & - \\
Patient dead & 3 & - \\
\hline
\end{tabular}


Table 3 Problems of hydroxyapatite orbital implantation and management in 250 consecutive cases

\begin{tabular}{|c|c|c|}
\hline Problem & Number & Management of problem \\
\hline Conjunctival thinning & 8 & $\begin{array}{l}\text { Adjust prosthesis vault and fit } \\
\text { Observation }\end{array}$ \\
\hline Conjunctival erosion ${ }^{\star}$ & 4 & $\begin{array}{l}\text { Adjust prosthesis vault and fit } \\
\text { Scleral patch graft and conjunctival flap }\end{array}$ \\
\hline Orbital haemorrhage & 0 & - \\
\hline Orbital infection & 1 & Intravenous antibiotics (implant retained) \\
\hline Implant migration & 0 & - \\
\hline Implant extrusion & 0 & - \\
\hline Peg extrusion & 2 & Remove granulation tissue in drilled peg hole \\
\hline
\end{tabular}

^ Conjunctival erosion due to flat or irregular vault on posterior surface of the prosthesis in three cases and poor surgical wound closure in one case.

Of the 250 cases, we considered 140 patients eligible candidates for peg placement (Table 2). Of the 140 eligible patients, 109 were satisfied with their cosmetic appearance and motility and preferred not to have the peg placed. A peg was successfully placed in the other 31 cases. We prefer to wait until the implant is 6 months from the time of placement to allow adequate fibrovascular ingrowth and we prefer the patient to be at least 6 years of age so that cooperation with the ocularist is adequate for ideal prosthesis revision. If these two criteria are not met then we consider the patient temporarily ineligible for peg placement.

Problems with the hydroxyapatite orbital implant were few and included conjunctival thinning without erosion in eight cases, conjunctival erosion in four cases, presumed orbital

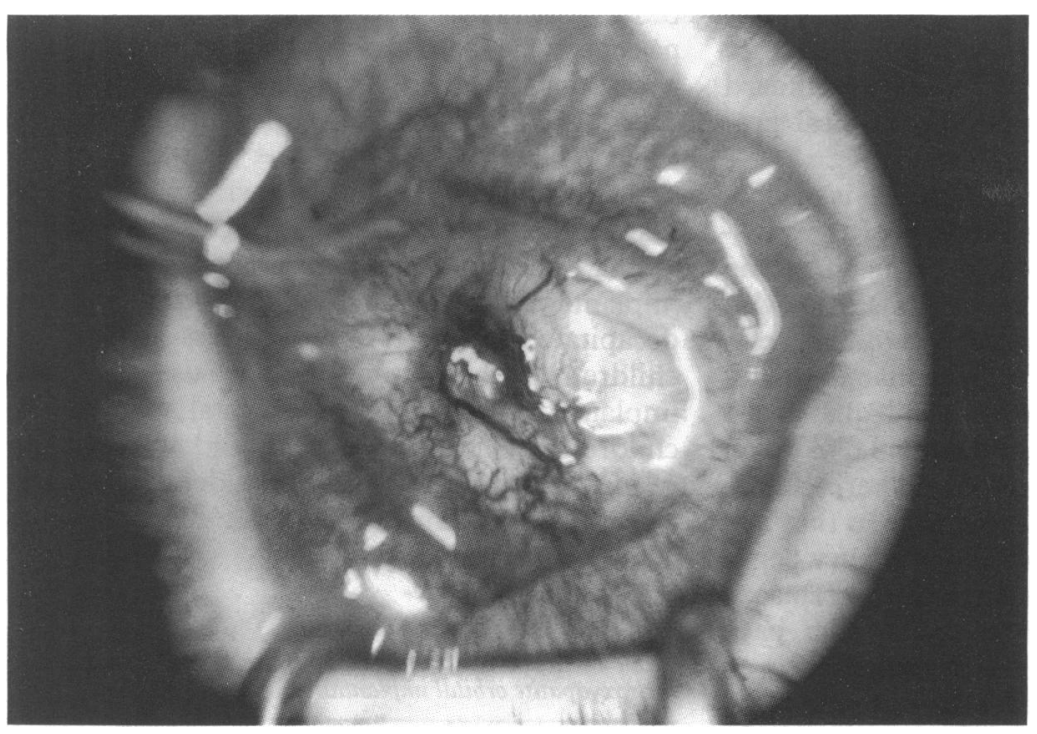

Fig $1 A$

Figure 1 (A) Late conjunctival erosion 7 months after hydroxyapatite placement in a child. (B) The prosthesis vault was flat and presumably caused pressure necrosis leading to the erosion ( $a$, high posterior prosthesis vault; $b$, flat irregular posterior prosthesis vault).

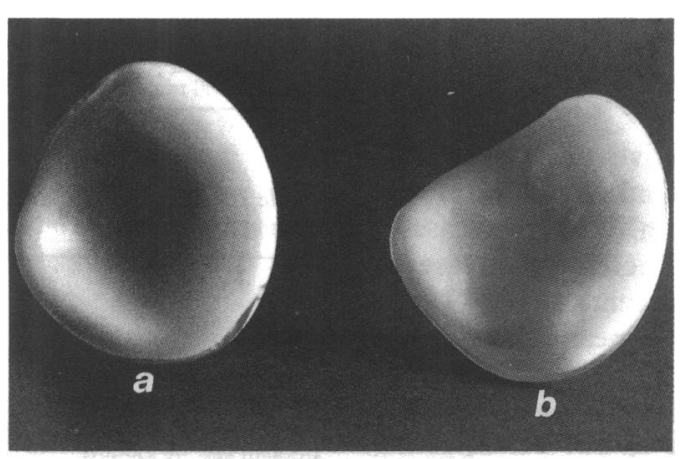

Fig $1 B$ infection in one case, and peg extrusion in two cases (Table 3). Five of the eight cases $(62 \%)$ of conjunctival thinning and three of the four cases $(75 \%)$ of conjunctival erosion occurred in children under 5 years of age. There were no cases of orbital haemorrhage, implant migration, or implant extrusion.

In all cases of conjunctival thinning the treatment was prosthesis adjustment and careful observation. In three of the four cases of conjunctival erosion the treatment was surgical repair and prosthesis adjustment. Those three cases had poorly fitting prostheses with pressure points on the conjunctival surface that led to the erosion 6, 7, and 12 months after enucleation, respectively. The erosion in these three cases measured approximately $4 \mathrm{~mm}$ in diameter and the underlying sclera was melted at the site of erosion. Two of the three cases were managed by the same ocularist and had a fairly flat posterior prosthesis vault that needed to be more highly vaulted (Fig 1). The last of the three cases developed erosion 12 months after enucleation and orbital magnetic resonance imaging showed deficient vascular ingrowth within the central portion of the implant (Fig 2). The fourth case of conjunctival erosion occurred 1 month after enucleation and was the result of inadequate wound closure. All cases of conjunctival erosion were repaired with conjunctival wound repair initially, three subsequently required a scleral patch graft/conjunctival flap for adequate closure.

The two cases of peg extrusion were first recognised at 1 and 7 months after peg placement. Both were caused by granulation tissue filling the orifice for the peg and pushing the peg out. Our recommendation was to remove the granulation tissue and replace the peg. In no cases did the peg simply fall out of its otherwise normal orifice. In three cases, there was a subtle but audible click of the peg on the back of the prosthesis in extreme gaze.

The case of presumed orbital infection, characterised by persistent mild pain and mucoid discharge, occurred 3 months after enucleation in a child with an upper respiratory illness. It was diagnosed and managed elsewhere and was found to be conjunctival swab culture negative and blood culture negative and resolved with intravenous antibiotics. The implant was retained within the orbit and the cosmetic appearance and motility of the prosthesis were undisturbed after treatment.

\section{Discussion}

Porous hydroxyapatite is a material derived from the skeletal structure of specific marine corals and it is composed primarily of calcium phosphate with a regular system of interconnecting pores of approximately $500 \mu \mathrm{m}$ diameter. ${ }^{17 \mathrm{l} 18}$ The porous nature of this substance encourages ingrowth of host fibrovascular tissue and converts this inert structure into living tissue, similar to bone. Hydroxyapatite has been used as a bone substitute in the fields of orthopaedics and maxillofacial and dental reconstruction for the past 15 years. ${ }^{17-23}$ The structure eventually transforms into woven and then lamellar bone over 
Figure 2 (A) Erosion of the conjunctiva overlying $a$ hydroxyapatite orbital implant 12 months after enucleation of a large choroidal melanoma in an adult. (B) Magnetic resonance imaging of orbit with gadopentate contrast demonstrated a central area within the orbital implant without enhancement (arrow), indicating poor implant vascularisation and possibly a collection of serous fluid. This may have contributed to the overlying tissue erosion.

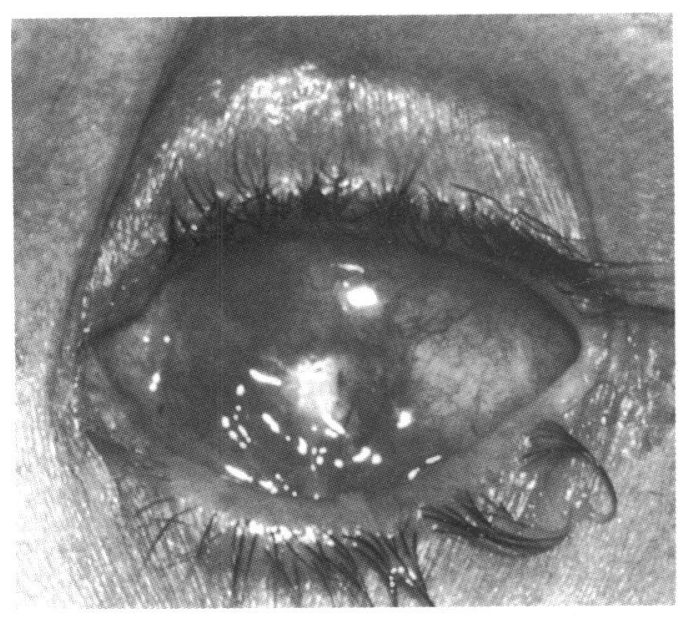

Fig $2 A$

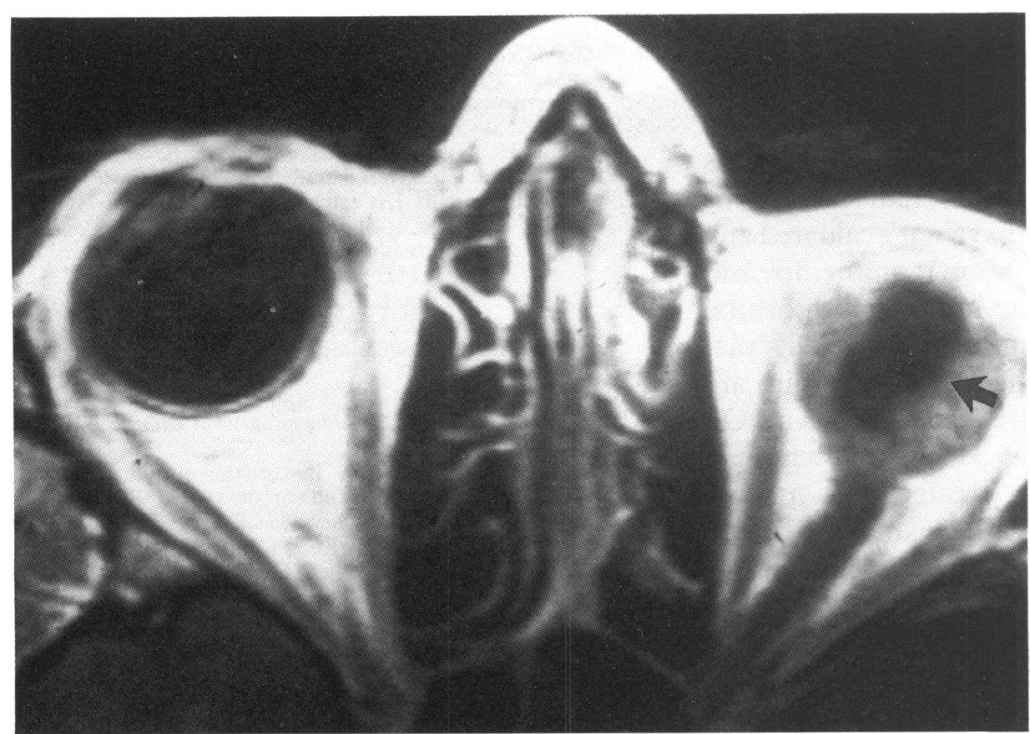

Fig 2B often shave the anterior portion of the hydroxyapatite sphere in order to alleviate the problem of conjunctival thinning and erosion. ${ }^{6}$

In assessing a patient with conjunctival thinning or erosion over an implant we believe that the length of time since enucleation may help identify the source of the problem and aid in management. If the defect is found in the first few weeks after enucleation then it is probably due to inadequate wound closure after digestion of the absorbable suture. The suture may need to be replaced if the defect is large. If the conjunctival defect occurs several months or later after the implant is placed it may be because of a poorly fitting prosthesis causing pressure necrosis, anterior orbital malplacement of the implant, inadequate closure of Tenon's fascia over the implant, or lack of implant vascularisation. In those patients with late conjunctival breakdown we first assess the fit of the prosthesis. If the posterior vault and edges of the prosthesis appear appropriate then we address the implant itself.

We prefer to manage conjunctival thinning conservatively by observation. In those sockets with conjunctival erosion we 'roughen' the edges of the overlying conjunctiva with a blunt instrument to encourage granulation tissue. If this does not close the defect satisfactorily over a several month period, then we revise the wound and anterior orbit by loosening all adhesions to the implant and reclosing the wound with generous Tenon's fascia and conjunctiva. If the defect persists despite this, then we dissect the tissue extensively and place a scleral patch graft if the implant sclera has melted. A generous Tenon's fascia closure and conjunctival flap are fashioned over the sclera. We have not had to exchange or remove any implant as a result of delayed healing of the conjunctiva.

We have not used the implant for eviscerated globes or after removal of infected globes, perhaps because most of our patients had intraocular tumours. One group reported that four of six patients who had hydroxyapatite implants after evisceration experienced tissue breakdown and exposure of the implant. ${ }^{24}$ The evisceration was performed for endophthalmitis in two cases. They also observed wound breakdown and implant exposure in four of 31 cases where the hydroxyapatite implant was used after enucleation. ${ }^{24}$ In three of the four cases the breakdown occurred 2 to 4 weeks after implant placement and we suspect this was due to inadequate tissue closure at the time of surgery as we observed in one of the cases that we report in this study. Fortunately, five of the eight wound breakdowns in their series healed with conservative methods without surgical intervention. ${ }^{24}$ Others have reported six cases of wound breakdown over the hydroxyapatite implant and these included two cases after evisceration, one after implant exchange in an previously infected socket, one after implant exchange in a socket that had extruded a silicone implant, and two after primary enucleation. ${ }^{25}$ It seems from these reports that hydroxyapatite is less well tolerated in eviscerated eyes and those eyes with earlier infection. Our study did not evaluate eviscerated eyes or eyes with endophthalmitis: however; we and the oculist's attention to vaulting the posterior surface of the prosthesis to prevent pressure necrosis. Although we originally did not modify the shape of the implant, we currently 
have found that patients who undergo enucleation for intraocular malignancies have an exceptionally good tolerance of the implant. Hydroxyapatite is well tolerated in both adults and children. ${ }^{26}$ We have used this material in 70 children enucleated primarily for retinoblastoma and it has not interfered with orbital examination. One can palpate the soft tissues around the implant to detect recurrence. Both hydroxyapatite and the previously employed polymethylmethacrylate appear densely radioopaque on computed tomography and with a low signal intensity on magnetic resonance imaging; therefore orbital imaging is the similar with the two implant materials. If retinoblastoma recurs in the orbit it is generally non-calcified, not radioopaque, and variable in its signal intensity so that the orbital implant should not interfere with its detection on computed tomography or magnetic resonance imaging.

One might suspect that prior radiotherapy would decrease fibrovascular ingrowth in the hydroxyapatite implant. We did not find an increased number of problems in those patients who had prior external beam radiotherapy or plaque radiotherapy. We have not evaluated any patients who had external beam radiotherapy and subsequent hydroxyapatite implant with orbital magnetic resonance imaging for implant vascularity. Most of our patients in this group are children who have not yet had peg placement. On the other hand, five of the 31 patients who had peg placement had been treated with plaque radiotherapy for a uveal melanoma before enucleation and all of these patients had adequate vascularity of the implant by magnetic resonance imaging. None of these five patients have had problems with their implant or peg.

It is comforting that most patients are satisfied with their motility and cosmetic appearance without peg placement. Even without the peg, the motion of the implant in the socket is often excellent since a portion of this motility is generally transferred to the prosthesis via conjunctival forniceal movement and conjunctival friction. ${ }^{7}$ Only 31 of the 140 eligible patients chose to have the peg placed. The problems of peg placement are few. Two patients had extrusion of the peg because of excessive granulation tissue that could be excised and repaired. There were no infections caused by the peg. Only one minor problem of a subtle audible peg click in and out of the ball and socket joint in extreme gaze was heard by three patients who had the peg in place. The sound was inaudible to anyone but the patient and was only heard by the patient when the gaze was extreme and the peg travelled further in the socket than the prosthesis could travel owing to the prosthesis size.

Hydroxyapatite orbital implant is well tolerated by all age groups with few problems. We suspect that the future will bring more improvements with this implant and the implant coverage. Fabricated mesh coverage rather than donor scleral coverage of the implant is being investigated. The eventual goal is to provide the patient with a well tolerated, moving artificial eye.

Presented at the American Ophthalmological Society, Hot Springs, Virginia on 24 May 1993.

Support provided by the Eye Tumor Research Foundation, Support provided by
Philadelphia, PA, USA.

1 Gougelmann HP. The evolution of the ocular motility implant. In: Shannon GM, Connelly FJ, eds. Oculoplastic surgery and prosthetics. Boston: Little, Brown, 1970: 689-711.

2 Kennedy RE. Enucleation, evisceration, and exenteration. In: Iliff NT, ed. Complications in ophthalmic surgery. New York: Churchill Livingstone, 1983: 487-513.

3 Soll DB. The anophthalmic socket. Ophthalmology 1982; 89: 407-23.

4 Perry AC. Advances in enucleation. Ophthal Plast Reconstr Surg 1991; 4: 173-7.

5 Perry AC. Integrated orbital implants. In: Bosniak SM, Smith $\mathrm{BD}$, eds. Advances in ophthalmic plastic and reconstructive surgery. Elmsford, NY: Pergamon Press, 1990: 75-81.

6 Dutton JJ. Coralline hydroxyapatite as an ocular implant. Ophthalmology 1991; 98 370-7.

7 Shields CL, Shields JA, De Potter P. Hydroxyapatite orbital implant after enucleation. Experience with initial 100 consecutive cases. Arch Ophthalmol 1992; 110: 333-8.

8 Shields JA, Shields CL. General principles of management. In Shields JA, Shields CL, eds. Intraocular tumors. A text and atlas. Philadelphia: WB Saunders, 1992: 37-41.

9 Shields CL, Shields JA, Eagle RC, De Potter P. Hydroxyapatite orbital implant. Histopathologic evidence of apatite orbital implant. Histopathologic evidence of Ophthalmol 1991; 111: 363-6.

10 De Potter P, Shields CL, Shields JA, Flanders AE, Rao VM Role of magnetic resonance imaging in the evaluation of the hydroxyapatite orbital implant. Ophthalmology 1992; 99: 824-30.

11 Shields JA, Shields CL, De Potter P. Enucleation technique for children with retinoblastoma. $\mathcal{f}$ Pediatr Ophthalmol Strabismus 1992; 29: 213-5.

12 Harbison MA, Hammer SM. Inactivation of human immunodeficiency virus by betadine products and chlorhexidine. deficiency virus by betadine products and chlor
f Acquired Imme Deficiency Synd 1989; 2: 16-20.

13 Benevento WJ, Murray P, Reed CA, Pepose JS. The sensitivity of Neisseria gonorrhea, Chlamydia trachomatis, and Herpes simplex type II to disinfection with povidone-iodine. Am $\mathcal{F}$ Ophthalmol 1990; 109: 329-33.

14 Merriam JC, Rosskothen HD, Benedek E. Right-angled peg for hydroxyapatite orbital implant. Ophthalmic Surg 1992 23: 564 .

15 Regillo CD, Shields CL, Shields JA, Eagle RC, Lehr J. Ocular tuberculosis. FAMA 1991; 266: 1490 .

16 De Potter P, Shields JA, Shields CL, Santos R. Modified enucleation via lateral orbitotomy for choroidal melanoma with orbital extension: a report of two cases. Ophthal Plast with orbital extension: a report

17 Piecuch JF. Extraskeletal implantation of porous hydroxyapatite ceramic. F Dent Res 1982; 61: 1458-60.

18 Piecuch JF, Topazian RG, Skoly S, Wolfe S. Experimental ridge augmentation with porous hydroxyapatite implants. f Dent Res 1983; 62: 148-54.

19 Kenney EB, Lekovic V, Sa Ferreira JC, Han T, Dimitrijevic B, Carranza FA. Bone formation within porous hydroxyapatite implants in human periodontal defects. $\mathcal{F}$ Periodomtol 1986; 57: 76-83.

20 Holmes RE. Bone regeneration within a coralline hydroxyapatite implant. Plast Reconstr Surg 1979; 63: 626-30.

21 Sartoris DJ, Gershuni DH, Akeson WH, Holmes RE, Resnick D. Coralline hydroxyapatite bone graft substitutes: preliminary report of radiographic evaluation. Radiology 1986; 159: 133-7.

22 White E, Shors EC. Biomaterial aspects of Interpore-200 porous hydroxyapatite. Dental Clin N Am 1986; 30: 49-67.

23 Rosen HM. Surgical correction for the vertically deficient chin Plast Reconstr Surg 1988; 82: 247-55.

24 Buettner H, Bartley GB. Tissue breakdown and exposure associated with orbital hydroxyapatite implant. $A m \mathcal{F} O p h-$ thalmol 1992; 113: 669-73.

25 Goldberg RA, Holds JB, Ebrahimpour J. Exposed hydroxyapatite orbital implants. Report of six cases. Ophthalmology 1992; 99: 831-6.

26 De Potter P, Shields CL, Shields JA, Singh AD. Use of the hydroxyapatite implant in the pediatric population. Arch Ophthalmol 1994; 112: 208-12. 